\title{
ICU quick drug guide: first edition
}

\author{
Jennifer Pai Lee (Editor); Elsevier, Philadelphia, PA, USA; price: CAD \$65.99 \\ (paperback); number of pages: 368; paperback. ISBN: 9780323680479
}

\author{
Najla Tabbara, PharmD (1) - Lisa Burry, PharmD
}

Received: 26 October 2020/Accepted: 27 October 2020/Published online: 16 November 2020

(C) Canadian Anesthesiologists' Society 2020

Because of the advances in evidence-based practice and increasing patient complexity, providing pharmaceutical care in a dynamic intensive care unit (ICU) can be challenging. Despite several published guidelines and drug handbooks, health care professionals lack a concise resource to obtain quick and accurate information regarding drug therapies. In its first edition, the ICU Quick Drug Guide provides a valuable overview of common disease states encountered in critically ill adults followed by the detailed pharmacotherapy principles applied when caring for these patients.

This book features six clinical sections: 1) Cardiovascular Critical Care; 2) Endocrine, Gastrointestinal, and Hepatic Disorders; 3) Infectious Diseases; 4) Neurocritical Care; 5) Pulmonary Disorders; 6) Miscellaneous. Each section is divided into three to seven chapters of varying lengths depending on the complexity of the topic-i.e., as short as six pages for the chapter covering diabetic ketoacidosis and the hyperosmolar hyperglycemic state and as long as 54 pages for the chapter describing all infections (with the exception of abdominal infections, which are addressed in the preceding chapter). Dr. Jennifer Pai Lee, the book's Editor, ensured that the chapters were organized into a consistent, easy-to-follow format that includes an overview of pathophysiology, diagnostics (including algorithms), goals of care, and management strategies (pharmacological and non-pharmacological).

As expected from the book's title, the highlight of this handbook is its presentation of detailed drug information. Prior to suggesting initiation of a new pharmacological intervention, the book reviews medication-induced causes of the conditions addressed and their possible mechanisms.

N. Tabbara, PharmD $(\bowtie) \cdot$ L. Burry, PharmD

Department of Pharmacy, Mount Sinai Hospital, Toronto, ON, Canada

e-mail: Najla.Tabbara@sinaihealth.ca
Reversible causes are also identified. This approach serves as an important reminder to clinicians that before prescribing additional medications to manage an acute condition, thorough assessment of current therapies may comprise effective patient management, thereby avoiding the possibility of prescribing cascades. The main features of each chapter are the medication tables that provide mechanisms of action and dosing (when applicable, the initial dosages, suggested titration, and target doses, as well as dosing considerations in patients with renal dysfunction and various renal replacement modalities). The tables also include pharmacokinetic and pharmacodynamic activities and important safety considerations such as contraindications and significant drug interactions. To ensure that readers are aware of the quality of the evidence presented, levels of evidence for recommendations are included when applicable.

An important benefit of the ICU Quick Drug Guide, compared with other critical care drug handbooks, is that medications are not listed alphabetically. Instead, they are presented within the appropriate disease-state chapter, thereby enhancing the clinical context for the reader. For specific medications, the chapter or Appendix includes conversion tables that provide guidance on, for example, how to transition from an intravenous to an oral diuretic during heart failure and suggestions for opioid conversions when switching opioid analgesics. There are also recommendations on peri-procedural/peri-operative drug management, such as when to hold anti-platelet agents prior to coronary artery bypass graft surgery. In terms of therapeutic drug monitoring, the Appendix summarizes therapeutic drug levels and when the levels of the drugs should be assessed. This information would be a handy resource for all health care professionals involved in patient care. A bonus of the ICU Quick Drug Guide is its digital version, which contains all the material from the paperback book and can be easily used on a mobile device. The eBook version can be at health care professionals' 
fingertips while they are carrying out their patient care activities, which significantly strengthens the book's overall value.

To encourage its use by more senior learners and established clinicians, future editions of this book might include chapters specifically focused on medication administration issues such as drug compatibility. It would also be useful to include the special considerations that must be addressed when treating unique, critically ill populations such as geriatric and obstetrical patients and those who require medication to be administered via feeding tubes or other non-traditional routes. This book mainly focuses on American guidelines but future editions might present other established international guidelines to compare management strategies (even if represented only by algorithms or abbreviated drug tables).
Overall, it is worthwhile to invest in the ICU Quick Drug Guide. It provides basic principles of disease management followed by detailed pharmacotherapy strategies and clinical pearls. It can be used in many health disciplines and by a wide spectrum of care providers, ranging from junior learners to established health care professionals in the ICU.

Disclosures None.

Funding statement None.

Editorial responsibility This submission was handled by Dr. Hilary P. Grocott, Editor-in-Chief, Canadian Journal of Anesthesia.

Publisher's Note Springer Nature remains neutral with regard to jurisdictional claims in published maps and institutional affiliations. 